Journal of

Synchrotron

Radiation

ISSN 0909-0495

Eugenia Pechkova ${ }^{a, b}$ and Claudio Nicolini $^{\mathbf{a}, \mathbf{b}}$

${ }^{\mathbf{a}}$ Fondazione ElBA, via delle Testuggini snc, I-00134 Rome, Italy, and ${ }^{\mathbf{b}}$ Nanoworld Institute, University of Genova, Corso

Europa 30, Genova, Italy

\section{Synchrotron radiation and nanobiosciences - introductory overview}

This issue represents a special issue on Synchrotron Radiation and Nanobiosciences resulting from the workshop which took place in Porto Conte, Sardinia, Italy, on 9-12 September 2004, within the framework of the Fondazione Elba-Max Planck Forum on Nanoscale Science and Technology occurring yearly since 1999, with Fondazione Elba and Max Planck Society as sponsors (see www.fondazione-elba.org). This forum was also co-sponsored by the ESRF (www.esrf.fr), the Nanoworld Institute (www.nwi.unige.it) and the European Round Table for Synchrotron Radiation and Free Electron Laser (Project IA-SFS). The emphasis was on current and future directions that are perceived to have impacts on fundamental research and industry. The aim of this issue is to provide the proceedings of the forum, introducing recent developments in the field of synchrotron radiation and nanobiosciences. This special issue addresses this new emerging field at the crossing of physical sciences and biological sciences with profound new insights into the fundamental processes of life and into the development of new synchrotron-based technology.

The stimulating Round Table on European Synchrotron Sources and Programs, which also took place at the meeting, pointed to a growing attention to synchrotron radiation and nanobiotechnologies and to the need of a better coordination of all infrastructure facilities in Europe. It is indeed our opinion that Europe, in order to achieve the required critical mass and competitiveness, must take further actions along the lines taken by CERN in Geneva by concentrating on a unique European Center with adequate facilities and manpower to fulfil the demands of 25 nations with their individual needs and expertise. 